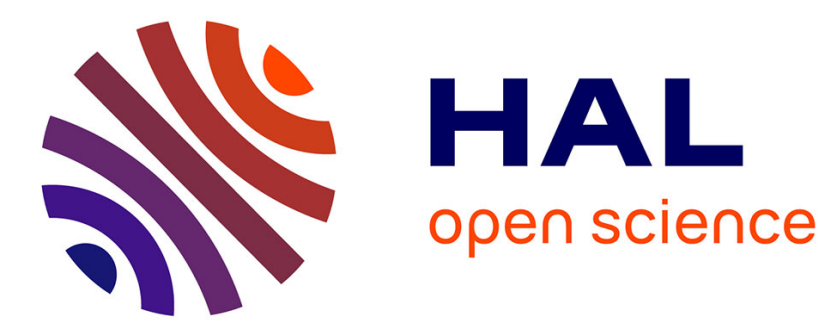

\title{
Quantitative thermal microscopy using thermoelectric probe in passive mode
}

Alexia Bontempi, Laurent Thiery, Damien Teyssieux, Danick Briand, Pascal Vairac

\section{- To cite this version:}

Alexia Bontempi, Laurent Thiery, Damien Teyssieux, Danick Briand, Pascal Vairac. Quantitative thermal microscopy using thermoelectric probe in passive mode. Review of Scientific Instruments, 2013, 84, pp.103703. 10.1063/1.4824069 . hal-00877408

\section{HAL Id: hal-00877408 \\ https://hal.science/hal-00877408}

Submitted on 28 Oct 2013

HAL is a multi-disciplinary open access archive for the deposit and dissemination of scientific research documents, whether they are published or not. The documents may come from teaching and research institutions in France or abroad, or from public or private research centers.
L'archive ouverte pluridisciplinaire HAL, est destinée au dépôt et à la diffusion de documents scientifiques de niveau recherche, publiés ou non, émanant des établissements d'enseignement et de recherche français ou étrangers, des laboratoires publics ou privés. 


\title{
Quantitative thermal microscopy using thermoelectric probe in passive mode
}

\author{
A. Bontempi, L. Thiery, D. Teyssieux, D. Briand ${ }^{*}$ and P. Vairac \\ FEMTO-ST, CNRS UMR 6174, 32 av. de l'observatoire, 25044 Besançon Cedex, France \\ "Ecole Polytechnique Fédérale de Lausanne, Institute of Microengineering, SAMLAB, Neuchâtel, Switzerland
}

\begin{abstract}
A scanning thermal microscope working in passive mode using a micronic thermocouple probe is presented as a quantitative technique. We show that actual surface temperature distributions of microsystems are measurable under conditions for which most of usual techniques cannot operate. The quantitative aspect relies on the necessity of an appropriate calibration procedure which takes into account of the probeto-sample thermal interaction prior to any measurement. Besides this consideration that should be treated for any thermal contact probing system, the main advantages of our thermal microscope deal with the temperature available range, the insensitivity to the surface optical parameters, the possibility to image DC and AC temperature components up to $1 \mathrm{kHz}$ typically and a resolution limit related to near-field behavior.
\end{abstract}

\section{INTRODUCTION}

Surface temperature measurement with high spatial resolution remains a major issue since none of the current techniques are mature enough to provide quantitative results. They can be classified in two groups: conventional and near-field. In any case, two uncertainties origin can be pointed out: the first is related to the physics principle of the technique and the second stems from the physics of heat transfer that relate the sample to the thermal detector.

Non-contact techniques are typical conventional techniques which are not subjected to the aforementioned uncertainties but are limited in terms of spatial resolution, temperature range and their dependence on the surface optical nature. These encompass optical techniques such as infrared or near-infrared thermography, ${ }^{1,2}$ thermoreflectance, ${ }^{3,4,5}$ photoluminescence $^{6}$ or Raman spectroscopy. ${ }^{7,8}$ Contact techniques overcome part of these drawbacks but thermal diffusion between the sample and the sensor becomes the main error source that needs to be carefully addressed. Among available techniques, liquid crystal thermography, ${ }^{9,10}$ fluorescence thermography ${ }^{11,12}$ or near-field optical thermography (NFOT) ${ }^{13,14}$ are also based on an optical measurement. However, the use of a probe tip ensures to increase the spatial resolution as it was demonstrated for all the near-field techniques. Thermal near-field microscopes are generally called Scanning Thermal Microscopes following the original idea of Williams and Wickramasinghe ${ }^{15}$ in 1986 that derived the use of a Scanning Tunnelling Microscope (STM) to operate on non-conducting samples. Progress in manufacturing thermal probes at the very tip of tapered materials have led to a family of near-field thermal 
techniques very closed to Atomic Force Microscope (AFM) for which a thermal sensitive area provides an additional information related to heat transfer between this area and the sample. The sensitive part can be quasi-punctual or distributed. Thermocouple or thermodiode allow a quasipunctual measurement while thermoresistive elements provide an integrated temperature value. These sensors are used to measure temperature distribution on hot samples (passive mode) or sample thermal properties when an additional heat source causes a heat transfer between the probe and the sample. The probe can be self-heated, typically by Joule effect (active mode) or an external heat source such as laser illumination. ${ }^{16}$ In any case, quantitative measurements remain hard to derive. Moreover, spatial resolution in thermal imaging is still an open issue since diffusion thermal process is depending on the probe dimension, but also the sample nature and the heated volume including the external condition (medium or vacuum) and the dynamics of the phenomenon (DC, AC or pulsed). Besides, temperature range of operation is fixed by the probe itself. Thermoresistive wire element such as Wollaston 5 micrometers in diameter platinum-rhodium wire probe is probably the most robust. ${ }^{17}$ Otherwise, thin-film microfabricated probes cannot exceed a hundred degrees typically except micro-pipette thermocouple for which the temperature limit is about $500^{\circ} \mathrm{C}^{18,19}$

For years, many attempts to develop micrometric size thermocouple or thermodiode sensors have been proposed by authors from the welding of $\mathrm{K}$ type wires, ${ }^{20} \mathrm{~S}$ types, ${ }^{21,22}$ thin-film deposition on a micropipette, ${ }^{23}$ to an $\mathrm{AFM}^{24,25}$ or cantilever structured ${ }^{25,26}$ or optical fiber tip. ${ }^{27}$ However, most of these developments remain inaccurate for quantitative measurements in ambient environment due to major contribution of probe-to-sample disturbing heat transfers. In this article, we focus on the passive mode of operation using a thermocouple probe which is fabricated with Wollaston wires of platinum and platinum-rhodium of 1.3 micron diameter welded together by means of a sparking technique. ${ }^{28}$ The chosen diameter represents a compromise between a maximum robustness and the minimum invasive effect of its contact. After a brief recall of the probe characteristics and the problematic of its use through the analysis of the thermal interaction with a sample, results of temperature scans on a microhotplate are presented as an actual alternative to available high resolution thermography.

\section{THERMOCOUPLE PROBE}

Accurate measurement in a large temperature range requires the use of standard bulk wires. However, available diameters are rather large, typically above 12 micrometers. The thinnest $\mathrm{K}$ type thermocouple wires are $7.6 \mu \mathrm{m}\left(3 \times 10^{-4}\right.$ inch). Wollaston wires remain the thinnest standard $\mathrm{S}$ type commercially available, whose fabrication process ensures to reduce a platinum or a $10 \%$ rhodiumplatinum core down to $0.5 \mu \mathrm{m}\left(2 \times 10^{-5}\right.$ inch $)$ inside a silver cladding. Among the different available diameters, $1.27 \mu \mathrm{m}\left(5 \times 10^{-5} \mathrm{inch}\right)$ represents the best compromise between size and robustness. The silver cladding is removed by a chemical or electro-chemical etching process so that a thermocouple junction can be obtained by welding platinum with rhodium-platinum wires. ${ }^{28}$ Sparking technique from a capacitor discharge is used. The next figure shows results of junction depending on the 
provided energy. Figure 1a shows the complete thermocouple for which the removed cladding length is about one hundred of micrometers. In figure $1 \mathrm{~b}$, wires temperature have reached their melting points due to an amount of energy of about $10 \mu \mathrm{J}$, leading to large balls on both wire extremities whereas in figure $1 \mathrm{~b}$, this phenomenon has been avoided by reducing the spark energy to about $4 \mu \mathrm{J}$.

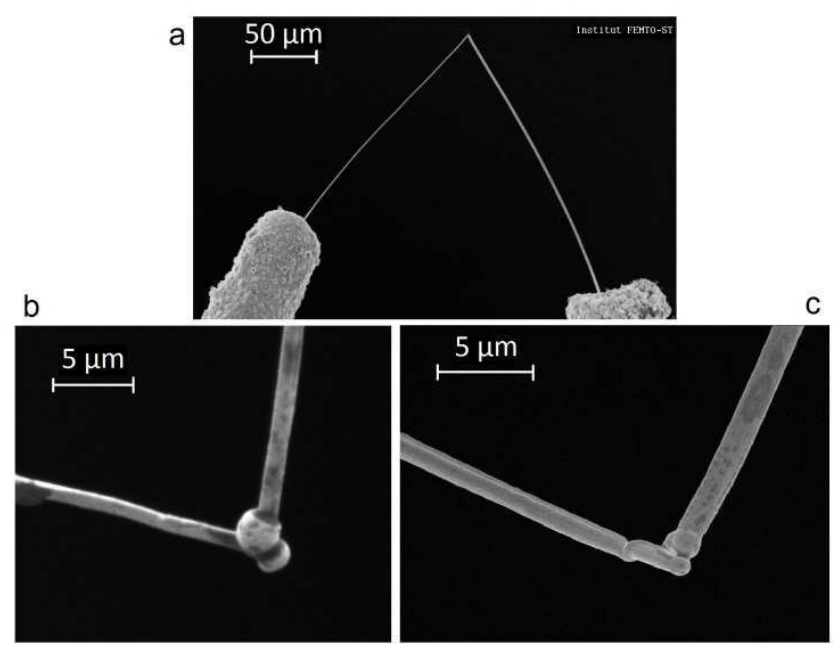

FIG. 1. Scanning electron micrograph of the thermocouple junction. General aspect (a), high energy sparking (b) and lower energy sparking (c).

Wollaston wires are commonly used as thermal resistors for hot-wire anemometry and commercial scanning thermal microscope (SThM®Topometrix), techniques for which the relation between the measured signal and the surface or contact temperature is hard to derive. Conversely, such a thermoelectric micro-junction provides punctual temperature signal that results from $\mathrm{S}$ type conversion law. Seebeck coefficient is known as a function of temperature, whose value is $5.88 \mu \mathrm{V} \mathrm{K}^{-}$ ${ }^{1}$ at $20^{\circ} \mathrm{C}, 9.13 \mu \mathrm{V} \mathrm{K}^{-1}$ at $300^{\circ} \mathrm{C}$ or $10.87 \mu \mathrm{V} \mathrm{K}^{-1}$ at $800^{\circ} \mathrm{C}$ as shown in figure 2.

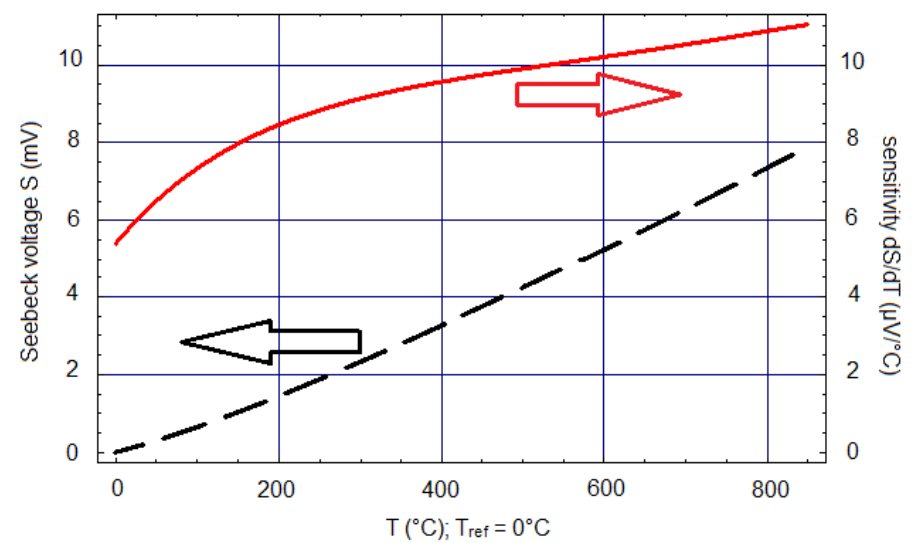

FIG. 2. S type thermocouple Seebeck voltage (dashed line) when reference temperature (cold junction) is $0^{\circ} \mathrm{C}$ and sensitivity as a function of temperature. 
The main reason that such probes are not widely used in laboratories is due to the difficulty to fabricate them reproducibly. However, by controlling the spark energy, the junction area remains close to the aspect of figure 1c. In addition, each thermocouple is tested according to a contact procedure previously described. ${ }^{29}$ Consequently, this probe remains a reliable tool for temperature mapping of microsystems especially for temperature ranging from ambient to $800^{\circ} \mathrm{C}$ typically and conditions for which optical techniques are not suitable.

\section{TIP TO SAMPLE THERMAL CONTACT}

The advantage of a microthermocouple regarding thermoresistive elements is the presence of a junction from which a Seebeck voltage relies to its temperature. Such a local temperature however never represents a true surface temperature since it results from a new thermal equilibrium. The main difference between contact and non-contact (optical) techniques results from the surface temperature disturbance. A suitable parameter to quantify the difference between actual and measured temperature is the probe thermal response $\tau$. It results from the expression of the thermal power that dissipates between sample surface and medium through the probe. Figure 3 depicts the thermal effect of such a heat dissipation $\mathrm{Q}$.

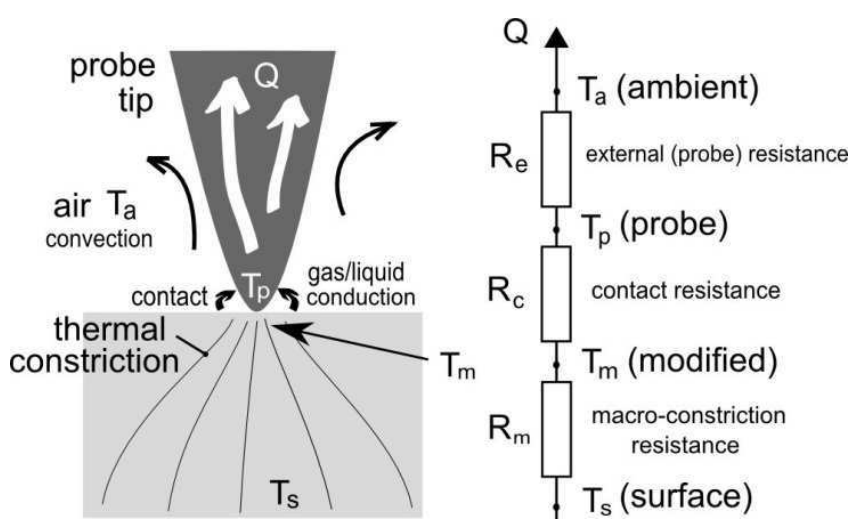

FIG. 3. Principle of thermal interaction between a probe tip and a hot surface.

Following figure 3 description, thermal power dissipation is expressed as:

$$
Q=\frac{T_{s}-T_{a}}{R_{m}+R_{c}+R_{e}}=\frac{T_{p}-T_{a}}{R_{e}}
$$

$\tau$ is deduced from the ratio between the probe temperature and the actual surface temperature relatively to ambient. Assuming constant values of thermal resistances in a large temperature range $\tau$ can be written as:

$$
\tau=\frac{T_{p}-T_{a}}{T_{s}-T_{a}}=\frac{R_{e}}{R_{m}+R_{c}+R_{e}}=\frac{d T_{p}}{d T_{s}}<1
$$


Such a definition takes into account the probe-to-surface thermal interaction as described in figure 3 in which the coupled effects of thermal transfers can be simply depicted as a series of thermal resistances. This suggests that depending on the sample nature and the ambient medium, one single probe thermal response may significantly vary. ${ }^{30,31}$ Different authors have used a more restrictive definition of a probe response assuming that the modification of internal thermal equilibrium of the sample could be negligible. ${ }^{32}$ This is realistic only when the sample is massive enough. In such a case, $\mathrm{R}_{\mathrm{m}} \rightarrow 0$ and $\mathrm{T}_{\mathrm{m}} \rightarrow \mathrm{T}_{\mathrm{s}}$ so that the mentioned parameter called $\phi$ is reduced to:

$$
\phi=\frac{R_{e}}{R_{c}+R_{e}}=\frac{T_{p}-T_{a}}{T_{m}-T_{a}}=\frac{d T_{p}}{d T_{m}}<1
$$

It could be considered as an intrinsic characteristic of the thermal probe whose ideal value would reach 1 in case of a perfect contact $\left(R_{c} \rightarrow 0\right)$. More generally, if $R_{e} \gg R_{c}$ any probe could be considered as efficient. We have measured these parameters in a previous paper for this temperature probe between ambient temperature up to $600^{\circ} \mathrm{C}$, leading to a mean value $\phi=0.934 .{ }^{29}$ In the same paper, it has been shown that thermal contact on thin samples such as micro-hotplate membranes could lead to noticeable effects of cooling down the point contact and changing slightly the thermal balance of the device. By measuring both the cooling down and the power of heat dissipated by the probe, the micro-hotplate thermal resistance $\mathrm{R}_{\mathrm{m}}$ has been estimated to $2.45 \times 10^{-4} \mathrm{~K} \mathrm{~W}^{-1}$. Then, the global thermal response of the probe was calculated as $\tau=0.925$. Such estimation is absolutely necessary for quantifying temperature measurements. However, another sample would imply another value of $\tau$ so that the following results have been obtained with a strictly identical sample device structure (see section 4). Among publications related to the mentioned contact techniques of temperature measurements, a very few have investigated this aspect of measurement quantification. In the very few data, one can mention Shi and Majumdar indications about their well-known batchfabricated thermocouple probe. ${ }^{30} \mathrm{~A}$ supposed asymptotic value $\phi=0.64$ has been obtained when measuring temperature on a $5.8 \mu \mathrm{m}$ width aluminum film. Reducing the film width demonstrated a decrease of this value down to 0.05 on a $0.35 \mu \mathrm{m}$ width. This clearly indicates an effect of cooling down due to the existence of non-negligible thermal resistance $\mathrm{R}_{\mathrm{m}}$ of the sample and that the mentioned value corresponds to the definition of $\tau$. This demonstrates that the use of $\phi$ ratio should be restricted to massive samples in order to compare different probes. Thermal response $\tau$ takes account of the sample nature and should be used to justify quantitative measurements. Furthermore, this also affects the lateral resolution $\Delta \mathrm{x}$ as proposed by the same authors. According to their definition, and our thermal response $\tau$, a corrected expression should give:

$$
\Delta x=\frac{\Delta T_{n}}{d T_{p} / d x}=\frac{\Delta T_{n}}{\tau \frac{d T_{s}}{d x}}
$$


where $\Delta \mathrm{T}_{\mathrm{n}}$ is the noise of the measurement chain and $\mathrm{dT}_{\mathrm{p}} / \mathrm{dx}$ is the measured lateral temperature gradient. According to this definition, lateral resolution is inversely proportional to the thermal response of the probe. As a result, only a perfect probe $(\tau \rightarrow 1)$ may exhibit the best lateral resolution. This also indicates that thermal lateral resolution does not depend on the probe only but also on the sample nature.

\section{EXPERIMENTAL SETUP}

A scanning thermal microscope is similar to near-field imaging system since the probe is a local tip that needs to be scanned over a sample. However, the contact is necessary to ensure an efficient thermal transfer between the surface and the tip. The contact force value depends on the tip geometry and its ability to support it without damage. Thin-wire thermocouples are flexible enough to undergo several tens of nano-Newton. Figure 4 depicts a distribution of measured DC temperature above the membrane center when approaching the probe from $1.8 \mathrm{~mm}$ to contact while the microhotplate is supplied with $13 \mathrm{~mW}$ DC power. DC thermocouple voltage is converted according to the Seebeck response of the S type standard law, corrected from ambient temperature (cold junction) and taking into account the effect of the probe thermal response given by expression (2) and quantified in reference 29, such:

$$
T_{s}=\frac{T_{p}-T_{a}}{\tau}+T_{a}
$$

The secondary graph of figure 4 represents the last 10 micrometers and the effect of the contact force increase between the thermocouple probe and the sample surface. Series of experiments are superimposed on this graph. Contact occurs at 0 abscissa and contact force increases for negative positions. Observed elastic deformation of the probe has allowed us to estimate that the maximum contact is in the range 50 to $100 \mathrm{nN}$. In this range, the measured temperature is stabilized showing that the surface to junction heat transfer is saturated. As a consequence, a precise control of the contact force is not necessary for this passive measurement mode. This is not the case in active mode of operation. ${ }^{33}$ It follows that our set-up remains quite simple without any contact control loop that would slow down the acquisition time. 


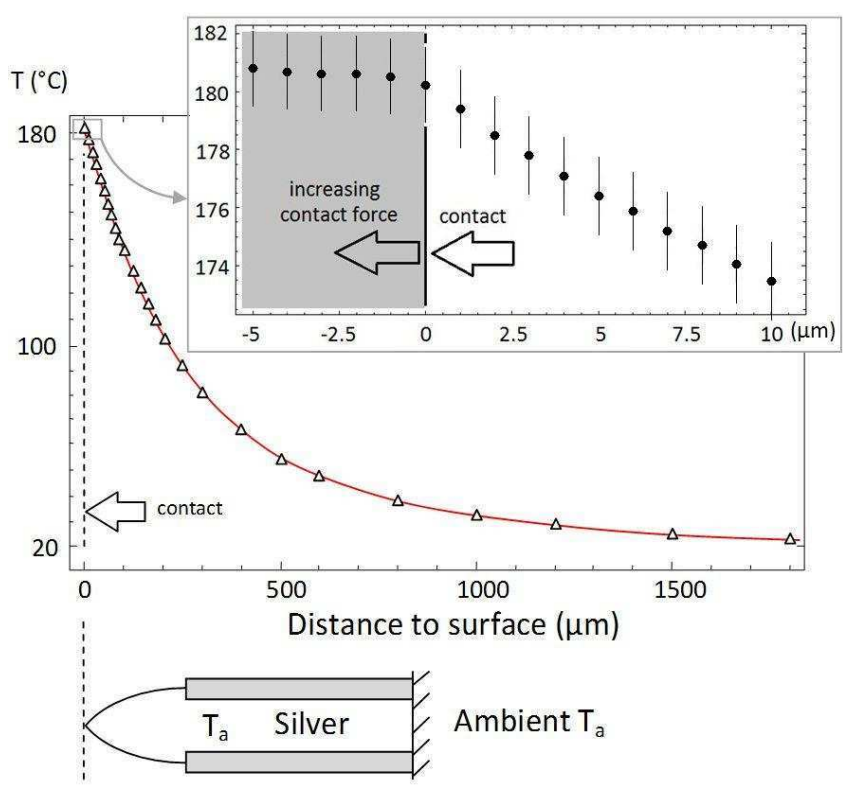

FIG. 4. Temperature gradient above the center point of the micro-hotplate up to $1.8 \mathrm{~mm}$ and schematic view of the probe at this scale. Influence of the contact force on the DC measured temperature value.

Measurements uncertainties are indicated on the secondary graph of figure 4. This represents the sum of usual $\mathrm{S}$ type thermocouple uncertainty $\left(1^{\circ} \mathrm{C}\right)$ and an estimated standard deviation obtained after 1000 successive approaches to contacts performed in the same condition of figure 4 , giving $0.3^{\circ} \mathrm{C}$. Besides, extracting absolute temperature of the thermoelectric junction requires knowing the reference temperature which corresponds to the separating area between the silver cladding and the platinum or platinum-rhodium wires. ${ }^{28} \mathrm{~A}$ large diameter silver wire acts as a perfect heat sink connected to ambient medium. This particular point is also depicted in the figure 4 with the geometry of the thermocouple probe in front of the sample surface and the temperature gradient. The length of the thermocouple wires is typically between 250 and $300 \mu \mathrm{m}$ and the silver cladding is $75 \mu \mathrm{m}$ of diameter. It has been verified that thermal conduction in silver is sufficient for ensuring a homogeneous ambient temperature. This value is measured by means of a thin-film platinum resistor (Pt1000) which is fixed in the vicinity of the silver wires.

Figure 5 presents a general view of the setup. Three piezo-actuators axis are used for the sample displacement whereas a long-range vertical axis is used to rough approach of the probe near sample surface. Since the contact force is not a controlled parameter and as long as surface sample is flat, a reference plan is acquired by contacting a few reference points before starting a complete automated scan. The thermocouple voltage is connected to a low-pass filtering voltmeter and a lock-in amplifier for simultaneous DC and AC measurements. AC current source is used to supply the tested sample and a computer both records the data while point-to-point displacement is managed. Measurements can be made in static or harmonic mode since the thermal bandwidth of the probe is larger than the 
micro-hotplate one (see Fig. 7). However, the main difference between thermal and other near-field microscope such as STM or AFM systems is related to a larger time constant that require point by point measurements during which a tip-sample thermal equilibrium is necessary. This time is typically on the order of one second even if the time constant of the probe itself is much lower. This is mainly depending on the sample and the overall conditions of heat dissipations.

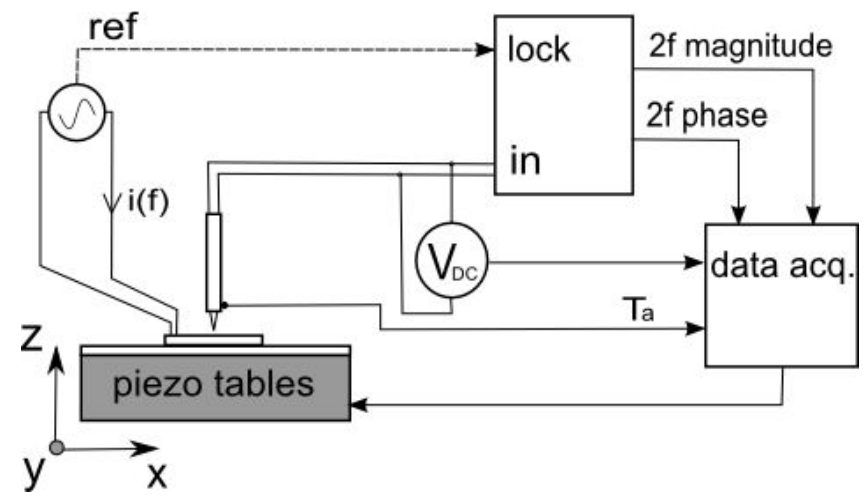

FIG. 5. Experimental set-up.

To demonstrate the performance of our system, micromachined silicon micro-hotplates represent pertinent electro-thermal devices to be studied since their active part is thermally insulated and can be heated up efficiently by Joule effect. The DC and/or AC current supplied to the metallic conductor generate DC and/or AC heating in the central active area. As shown in figure 6, the chosen device is similar to those which were described in previous papers and with which our probe has been calibrated $^{2,29}$. A $200 \mathrm{~nm}$-thick platinum film is embedded in a LPCVD silicon nitride suspended membrane whose thickness is $1 \mu \mathrm{m}$. The design of the platinum heater and the membrane thickness and nature are optimized to insure a homogeneous temperature distribution and the lowest power consumption. As shown in the results section, $20 \mathrm{~mW}$ is enough to reach $250^{\circ} \mathrm{C}$ typically near the central part of the membrane.

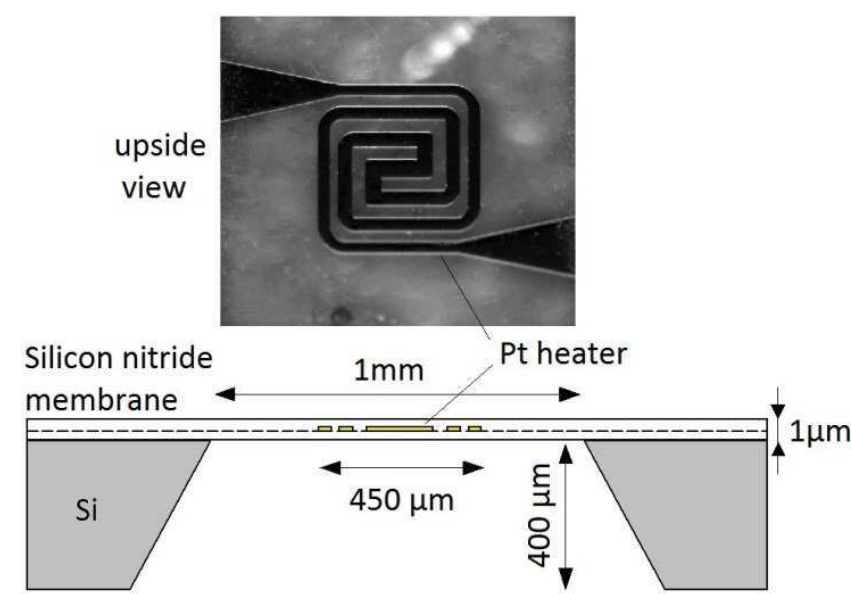

FIG. 6. Top view image and schematic of the cross-section of the micro hotplate. 


\section{RESULTS}

Depending on the excitation mode of the active sample, several thermal signals can be extracted from the thermoelectric probe voltage. A static current generates a DC heating and AC current at a frequency $f$ generates both a DC and a doubled frequency $2 \mathrm{f}$ heating of the heater due to Joule effect. A comparison between the thermal pass band of the sensor and micro-hotplate is however required before any measurement. Figure 7 presents the normalized magnitude of both the probe and the tested device while subjected to AC current at frequency f. Joule effect generates a double frequency $2 \mathrm{f}$ temperature elevation and a subsequent resistance variation that can be detected at the triple frequency $3 \mathrm{f}$ by means of a lock-in. Indeed, the measured voltage represents the product between the electrical resistance which varies at $2 \mathrm{f}$ and the supplied current at $\mathrm{f}$ frequency. This reveals thermal cut-off frequencies near $20 \mathrm{~Hz}$ for the micro-hotplate and $300 \mathrm{~Hz}$ concerning the thermocouple probe wires.

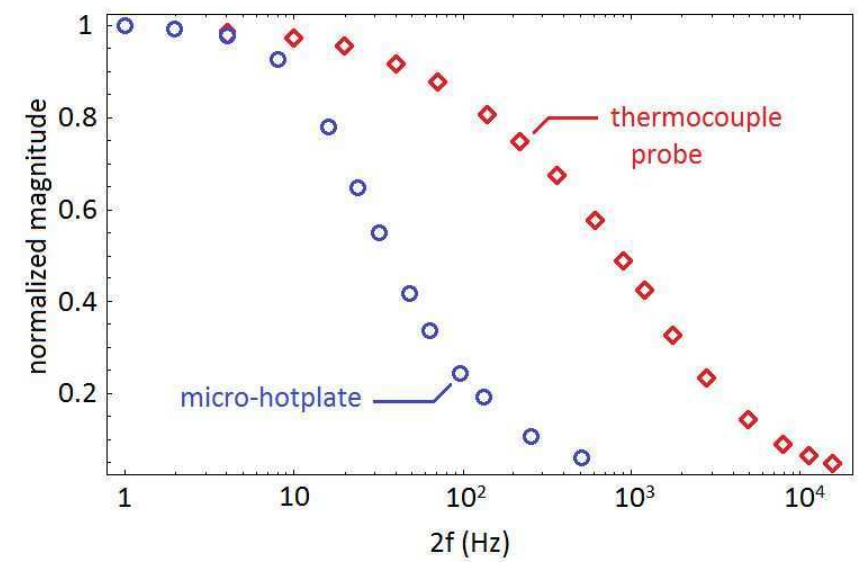

FIG. 7. Thermal pass band comparison between the thermocouple probe and the micro-hotplate.

Series of measurements have been performed while supplying an alternative current at a frequency f. As a result, three thermal signals are provided by the thermocouple using a DC voltmeter and a lockin (magnitude and phase) at the double frequency $2 \mathrm{f}$. The thermocouple probe has been scanned over the micro-hotplate and each measurement point has been stored after one second of contact. AC RMS voltage is converted in AC RMS temperature according to the sensitivity (derivative) of the S type thermocouple shown in figure 2 which is taken for each point at the probe DC temperature value $\left(T_{p}\right)$. The whole membrane is $1 \mathrm{~mm}$ square. Figure 8 presents a result of a complete scan while the heater is supplied with an AC RMS current of $10 \mathrm{~mA}$. DC temperature component points out the thermal homogeneity of the central area whereas AC $2 \mathrm{f}$ components reveal interesting thermal contrasts both in magnitude and phase. Despite the frequency range which exceeds the probe and the sample bandwidths, signal to noise ratio remain sufficient for extracting the following images. However, if DC images are quantitative, AC contrast provides only qualitative data. 
optical upside view

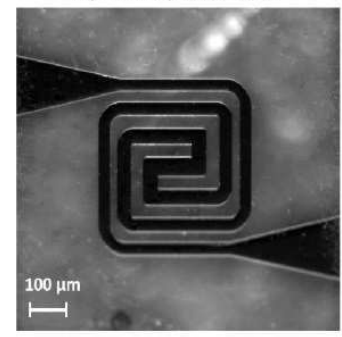

2f temperature phase; $f=270 \mathrm{~Hz}$

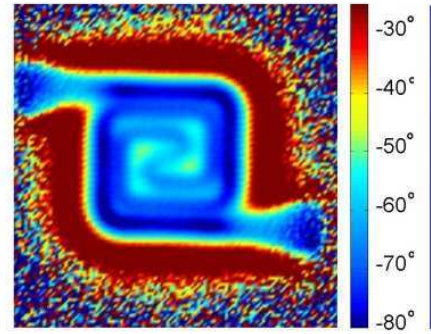

DC temperature component

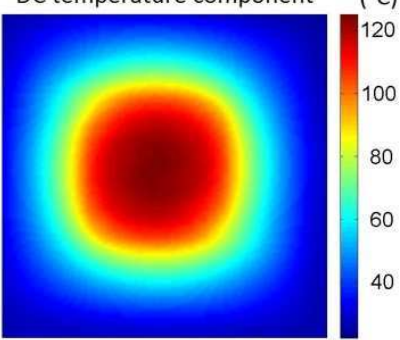

2f RMS magnitude; $f=270 \mathrm{~Hz} \quad\left({ }^{\circ} \mathrm{C}\right)$

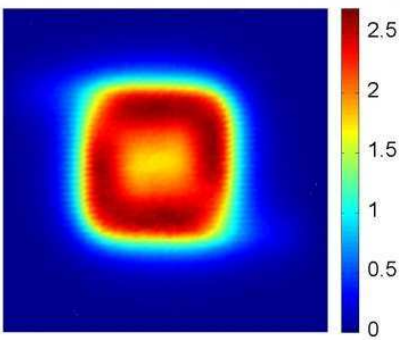

FIG. 8. Complete membrane scan $\left(106 \times 106\right.$ points $\left./ 1 \mathrm{~mm}^{2}\right)$ at a supply current frequency of $270 \mathrm{~Hz}$.

Let us recall that the platinum heater is only visible on the optical view due to the transparency of the silicon nitride membrane in which it is embedded. The phase signal clearly reveals its presence whereas magnitude points out the heat source intensity. The center of the coil being less resistant, the magnitude is consequently lower than for the perimeter area. The influence of the current frequency helps to reveal these details as presented in figure 9 where scans have been reduced to the central area (560 $\mu \mathrm{m}$ square). AC RMS supplied current is $12 \mathrm{~mA}$ and frequency varies between 10 and $850 \mathrm{~Hz}$. 


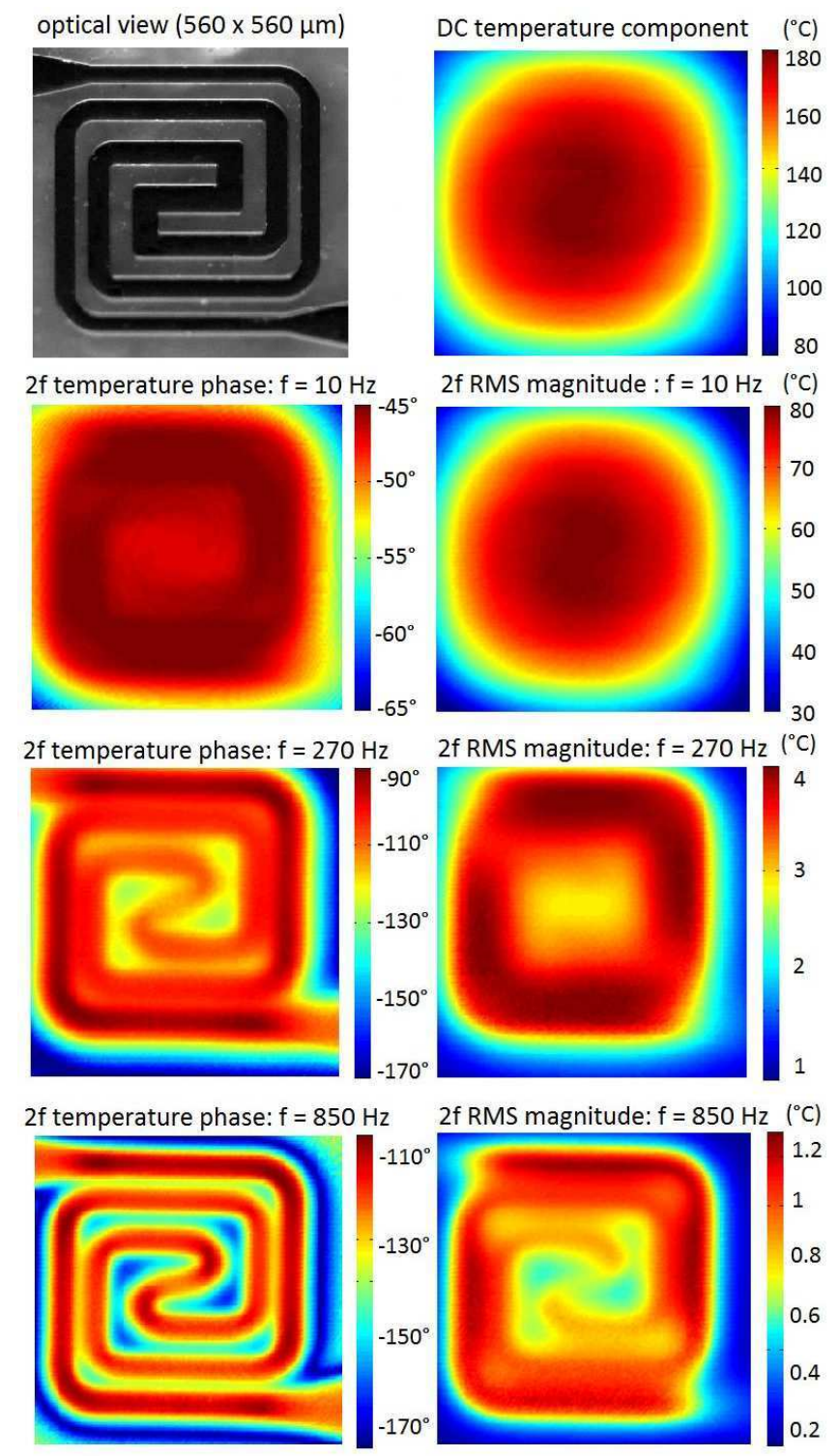

FIG. 9. Active part scan $(141 \times 141$ points / 560 x $560 \mu \mathrm{m})$ at different supply current frequencies.

Result on DC temperature distribution remains identical at any frequency. Besides, only the central part of the coil is slightly visible and comparable to $10 \mathrm{~Hz}$ frequency magnitude image. The heater geometry appears in both magnitude and phase images when increasing frequency. However, due to both micro-hotplate and the thermocouple pass band, magnitude decreases whereas phase contrast increases. Magnitude strongly depends on the dissipated power but phase only depends on the physics of heat transfers. Indeed, phase results from a competition between Joule heat source areas which exhibit phase values close to heat source and heat diffusion into materials. This is why the complete metallic coil is clearly visible at higher frequencies regarding to magnitude images. Conversely the silicon nitride membrane acts as a passive material which undergoes strong temperature gradients and strong phase variations. Thermal imaging remains strongly different to other near-field imaging system in the fact that the measured surface contrast derives from a volumetric contribution of heat 
diffusion's law. As a result, thermal contrast of these images is often considered as subsurface contrast.

\section{CONCLUSION}

We have shown that a standard micro-thermocouple can be used as a local temperature probe in a scanning quantitative thermal imager. In spite of a scanning operation that requires one second typically per acquisition point, this represents a real alternative to conventional techniques which are unable to operate from ambient to $800^{\circ} \mathrm{C}$ while remaining insensitive to surface optical nature. Besides, the quantitative aspect of these contact measurement represent a major point which has not been correctly addressed by authors yet whereas it represents the main limiting factor of local thermal probing. In spite of recent attempts to correct the effect of a probe contact ${ }^{34,35}$, quantitative measurements still remain generally inaccurate due to the dependence on the sample nature itself which is rarely considered. This is mainly due to the confusion between the actual surface temperature underneath the tip in contact and the actual temperature far beyond contact. The latter is the only relevant value whereas most of techniques do not mention any difference since the sample nature introduced with $\mathrm{R}_{\mathrm{m}}$ thermal resistance (see Fig.3) is neglected. According to our analysis and without taking account of artifacts due to topography, temperature quantification at micro and nano-scales is only possible if:

- The sample is already thermally characterized such as in the present paper $\left(\mathrm{R}_{\mathrm{m}}\right)$, leading to a correction of temperature measurements using $\tau,{ }^{29}$

- A relevant method for correcting the effect of contact by canceling the heat flux is applied (null point method). ${ }^{34}$

In such a case however, operating under vacuum is helpful because attempts to reduce only heat transfer at contact jump as proposed by Chung ${ }^{34}$ may not be sufficient to take account of the heat which is transferred during far approach of the probe. Indeed, as shown in figure 4, DC heat diffusion through air by means of conduction and convection affects measurements in a millimeter range from the sample surface. Moreover, this also affects the resolution limit which is not studied in this paper. This will be the subject of further articles as well as the extension of the present system to active mode of operation. ${ }^{33}$

To date, the very few tested nano-thermocouples which were fabricated on a silicon nitride tip similarly to AFM cantilevers and probably most of resistive thermal nano-probes are supposed to exhibit a response $\tau$ on the order of 0.05 when scanning nanostructured heated elements. ${ }^{30}$ Improving thermal characteristics of these probes would benefit to both resolution and a better evaluation of the quantification errors in surface temperature measurement. A common approach in scanning thermal microscopy would help to define measurable thermal quantities, including a standard definition of lateral resolution. Otherwise, no progress in thermal measurements at micro and nano-scales may be 
expected as long as micro-thermal probes will not be tested and compared in standard benchmark conditions.

\section{REFERENCES}

1. D. Teyssieux, L. Thiery and B. Cretin, Rev. Sci. Instrum. 78, 034902 (2007).

2. D. Teyssieux, D. Briand, J. Charnay, N. F. de Rooij and B. Cretin, J. Micromech. MicroEng. 18, 065005 (2008).

3. G. Tessier, S. Holé, and D. Fournier, Appl. Phys. Lett. 78, 2267 (2001).

4. J. Christofferson and A. Shakouri, Rev. Sci. Instrum. 76, 024903 (2005).

5. M. Farzaneh, K. Maize, D. Lüerssen, J.A. Summers, P.M. Mayer, P.E. Raad, K.P. Pipe, A.

Shakouri, R.J. Ram and J.A. Hudgings, J. Phys. D: Applied Physics 42 (14) 143001 (2009).

6. C. Herzum, C. Boit, J. Kölzer, J. Otto and R. Weiland, Microelec. J. 29, 163-170 (1998).

7. M. Kuball, S. Rajasingam, A. Sarua, M.J. Uren, T. Martin, B.T. Hughes, K.P. Hilton and R.S.

Balmer, Appl. Phys. Lett. 82 124-6 (2003).

8. A. Soudi, R.D. Dawson and Y. Gu, ACS Nano 5, 1, 255-262 (2011).

9. A. Csendes, V. Szekely and M. Rencz, Microelec. Engin. 31, 281-290 (1996).

10. C.K. Lau and K.S. Sim, AIP Conf. Proc. 1528, pp. 231-236, 2012 Nat. Phys. Conf. (PERFIK 2012) 19-21 nov, Malaysia.

11. E. Saidi, B. Samson, L. Aigouy, S. Volz, P. Löw, C. Bergaud and M. Mortier, Nanotechnology 20, 115703 (2009).

12. F. Vetrone, R. Naccache, A. Zamarron, A. Juarranz de la Fuente, F. Sanz Rodriguez and L. Martinez Maestro, ACS Nano 4 (6), 3254-3258 (2010).

13. K. E. Goodson and M. Asheghi, Microscale Thermophys. Eng. 1, 225-235 (1997).

14. T. Fuji, Y. Taguchi, T. Saiki and Y. Nagasaka, Rev. Sci. Instrum. 83, 124901 (2012).

15. C.C. Williams and H.K. Wickramasinghe, Appl. Phys. Lett. 49, 1587 (1986).

16. M. Stopka, L. Hadjiiski, E. Oerterschulze and R. Kassing, J. Vac. Sci. Technol. B, 13, 2153-56 (1995).

17. R.B. Dinwiddie, R.J. Pylkki and P.E. West, Thermal Conductivity 22, ed. T.W. Tong (Lancaster, PA:Technomics) p.668 (1994).

18. G. Fish, O. Bouevitch, S. Kolotov, K. Lieberman, D. Palanker, I. Turovets and A. Lewis, Rev. Sci. Instrum. 66 (5), 3300-3306 (1995).

19. Y. Zhang, P.S. Dobson and J.M.R. Weaver, J. Vac. Sci. \& Tech. B 30 (1) 010601 (2012).

20. A. Majumdar, J. Lai, M. Chandrachood, O. Nakabeppu, Y. Wu and Z. Shi, Rev. Sci. Instrum. 66 (6), 3584-3592 (1995).

21. R. A. Secco and R. F. Tucker, Rev. Sci. Instrum. 63 (11), 5485-5486 (1992).

22. L. Thiery, Rev. Gen. Therm. 394 (1994), 551-561. 
23. Yo. Zhang, Ya. Zhang, J. Blaser, T.S. Sriram, A. Enver and R.B. Marcus, Rev. Sci. Instrum. 69 (5), 2081-2084 (1998).

24. T. Leinhos, M. Stopka, E. Oesterschulze, Appl. Phys. A 66, S65 (1998).

25. G. Mills, H. Zhou, A. Midha, L. Donaldson and J.M.R. Weaver, Appl. Phys. Lett. 72 (22), 29002902 (1998).

26. L. Shi, O. Kwon, A.C. Miner and A. Majumdar, J. MicroElectroMechanical Sys. 10 (3), 370-378 (2001).

27. L. Thiery, C. Bainier, M. Spajer, OPTO 2002 International Conference Proceedings, 193-196, Erfurt (Germany) 14-16 mai 2002.

28. P. Voisin, L. Thiery and G. Bröm, Eur. Phys. J. Appl. Phys. 7 (2), 177-187 (1999).

29. L. Thiery, S. Toullier, D. Teyssieux, D. Briand, J. Heat Transfer 130, 091601 (2008).

30. L. Shi, A. Majumdar, J. Heat Transfer, 124, 329-332 (2002).

31. S. Gomes, N. Trannoy and P. Grossel, Meas. Sci. Technol. 10, 805-811 (1999).

32. L. Shi, S. Plyasunov, A. Bachtold, P.L. Mc Euen and A. Majumdar, Appl. Phys. Lett. 77 (26), 4295-4297 (2000).

33. L. Thiery, E. Gavignet and B. Cretin, Rev. Sci. Instrum. 80, 034901 (2009).

34. J. Chung, K. Kim, G. Hwang, O. Kwon, S. Jung, J. Lee, J.W. Lee and G.T. Kim, Rev. Sci. Instrum. 81, 114901 (2010).

35. F. Menges, H. Riel, A. Stemmer and B. Gotsmann, NanoLett. 12, 596-601 (2012). 